This item was submitted to Loughborough's Research Repository by the author.

Items in Figshare are protected by copyright, with all rights reserved, unless otherwise indicated.

\title{
Theoretical analysis on needle-punched carbon/carbon composites
}

PLEASE CITE THE PUBLISHED VERSION

https://doi.org/10.1007/s10443-018-9749-2

PUBLISHER

(C) Springer

VERSION

AM (Accepted Manuscript)

PUBLISHER STATEMENT

This is a post-peer-review, pre-copyedit version of an article published in Applied Composite Materials. The final authenticated version is available online at: https://doi.org/10.1007/s10443-018-9749-2.

\section{LICENCE}

CC BY-NC-ND 4.0

\section{REPOSITORY RECORD}

Han, Meng, and Vadim Silberschmidt. 2019. "Theoretical Analysis on Needle-punched Carbon/carbon Composites”. figshare. https://hdl.handle.net/2134/38102. 


\title{
Theoretical Analysis on Needle- Punched Carbon/Carbon Composites
}

\author{
Meng $\operatorname{Han}^{1,2} \cdot$ Vadim V. Silberschmidt ${ }^{2}$
}

\begin{abstract}
Needle-punched carbon/carbon composites (NP-C/Cs) are advanced materials widely used in aerospace applications. The needle-punching technique improves the integrality of carbonfibre plies, however, it also introduces many defects, affecting the mechanical behavior of NP$\mathrm{C} / \mathrm{Cs}$. A theoretical model of irregular beams is suggested to investigate the mechanical behavior of unidirectional needle-punched carbon/carbon composites. Stress distributions in punched and squeezed fibres and an effect of the needle-punching technology are assessed.
\end{abstract}

Keywords Carbon/carbon composites $\cdot$ Mesomechanics $\cdot$ Theoretical analysis

\section{Introduction}

Carbon/Carbon composites (C/Cs) demonstrate excellent properties such as high specific stiffness and strength, low density, good wear resistance, high thermal resistance and low thermal expansion [1]. As a result, they are widely used in aerospace structures and braking systems.

The needle-punched (NP) structure, produced from fibre fabrics and nonwoven webs with a through-thickness needling technique, has advantages of the combined high inter-laminar properties and lower-cost processing $[2,3]$. The NP C/Cs are prepared from the preforms by employing a chemical vapor-deposition (CVD) process, with heat-treatment at temperature of $2000 \mathrm{~K}$ for $2 \mathrm{~h}$, followed by densification at $1300 \mathrm{~K}$ under the pressure of $1 \mathrm{kPa}$. Thanks to the NP process, interlaminar properties are strengthened; however, many defects are introduced into laminates inevitably.

Some efforts were taken to characterise the performance of C/Cs. Zhang et al. [4, 5] investigated bending properties and fracture mechanism of $\mathrm{C} / \mathrm{Cs}$ with high-density preform and observed

Meng Han: hanmengmaker@163.com

1 State Key Laboratory of Mechanics and Control of Mechanical Structures, Nanjing University of Aeronautics and Astronautics, Nanjing 210016, People's Republic of China

2 Wolfson School of Mechanical, Electrical and Manufacturing Engineering, Loughborough University, Loughborough, Leicestershire LE11 3TU, UK 
delamination and interlaminar fracture. Li et al. [6-8] examined macro-fracture with scanning electron microscope (SEM) micrographs to understand the bending deformation and failure mechanism of 3D NP C/Cs at room and high temperatures. The obtained results showed that the loaddeflection curves below $400^{\circ} \mathrm{C}$ exhibited a linear elastic behaviour and brittle fracture, while failure above $500^{\circ} \mathrm{C}$ was plastic. Static compressive behavior of such composites was studied by Zhang et al. [9, 10], demonstrating that both transverse and longitudinal compressive strengths for materials with dual matrix were higher than those with a single matrix. The failure modes under transverse and longitudinal compressive loading regiones were shear and extension failure, respectively. Cai et al. [11-13] also investigated the bending properties of the 3D NP C/Cs. measuring the flexure strength of $98 \mathrm{MPa}$. Variety researchers [14-16] investigated effect of holes due to needling, e.g. properties of 3D z-pinned composites, including development model of their internal geometry and characterization of mechanical properties under static loading conditions.

Still, to the best of authors' knowledge, researches employing theoretical models and numerical simulations of mesoscopic features of $\mathrm{NP} \mathrm{C} / \mathrm{Cs}$ are rare. In this paper, the microscopic stress distributions are derived analytically for fibres and matrix; bending behavior of such composites is studied theoretical. Finally, some conclusions are drawn based on the obtained results.

\section{Mesoscopic Features of NP C/Cs}

The NP C/Cs studied in this paper were alternately stacked with $0^{\circ}$ and $90^{\circ}$ unidirectional (UD) carbon plies, as well as layers of short-cut-fibre web clothes between them, as schematically shown in Fig. 1(a). The composites contained ten layers and the thickness of each layer was $0.5 \mathrm{~mm}$.

The preform $[17,18]$ was punched by regularly arranged needles on the needle plate. A distance between the neighboring needles was $2.4 \mathrm{~mm}$ along the $\mathrm{X}$ direction and $4 \mathrm{~mm}$ along the $\mathrm{Y}$ direction (Fig. 1(b)), the diameter of needle hole is $0.9 \mathrm{~mm}$.

\section{Theoretical Analysis of UD NP C/Cs}

\subsection{Mesoscale Mechanical Model of Squeezed Fibre Bundles}

Fibres of UD NP C/Cs are squeezed in the X-Y plane as a result of application of the needlepunching technology $[19,20]$. To simplify the analysis, the produced hole shape formed by squeezed fibres is considered as the irregular hexagon (Fig. 2). This is used as a representative

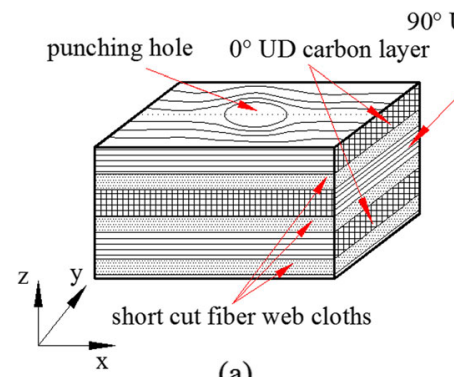

$90^{\circ} \mathrm{UD}$ carbon layer

(a)

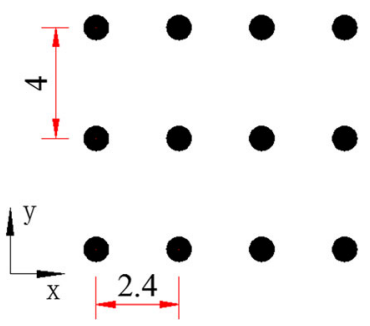

(b)

Fig. 1 Schematic diagram of geometry model: a NP C/Cs; b needle distribution 
volume element (RVE) for analysis below. Hence, the shape equation for the matrix can be suggested as:

$$
h_{m}(x)=\left\{\begin{array}{l}
\frac{r}{l} x(0<x \leq l) \\
r(l<x \leq(l+r))
\end{array}\right.
$$

where $r$ is the radius of the needle hole, $l$ is the range of the punching effect.

The shape equation of a fibre bundle has the following form:

$$
H(x)= \begin{cases}r \frac{x}{l}+\frac{h_{y}}{2} & (0 \leq x<l) \\ r+\frac{h_{y}}{2} & (l \leq x<l+r)\end{cases}
$$

where $h_{y}$ is the height of fibre bundles before punching.

According to the relationship of deformation between the fibre bundles and the matrix (Fig. 3) [21-23], the shear strain of the latter can be presented as:

$$
\gamma_{m}=\frac{H(x)}{h_{m}(x)} y^{\prime}(x)
$$

where $h_{m}$ is the height of matrix, $H$ is the sum height of fibre bundle and matrix.

So, the shear stress of the matrix can be solved as:

$$
q_{m}(x)=\tau_{m}(x)=G_{m} \gamma_{m}=G_{m} \frac{H(x)}{h_{m}(x)} y^{\prime}(x)
$$

where $G_{m}$ is the shear modulus of matrix.

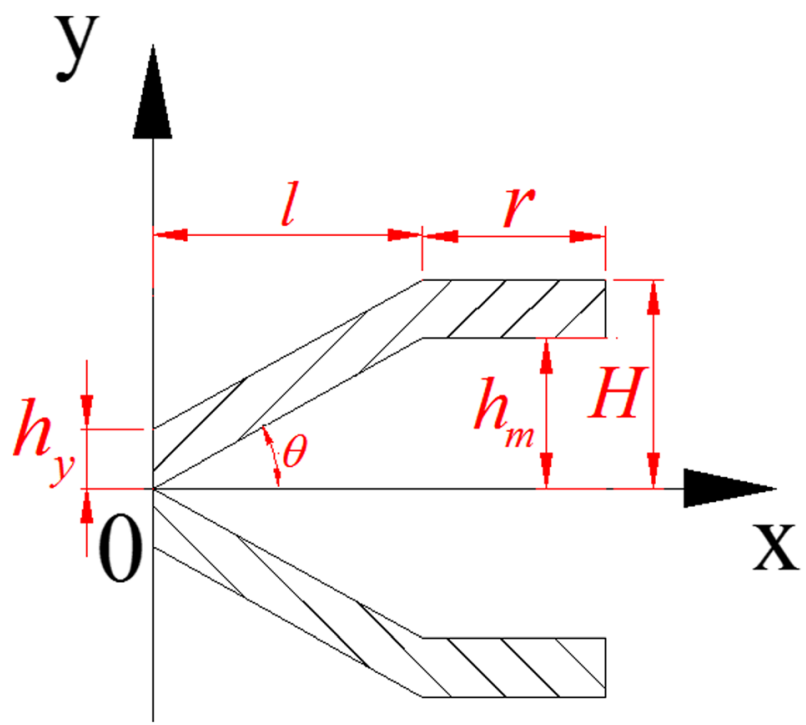

Fig. 2 Half of RVE of squeezed fibre bundles 


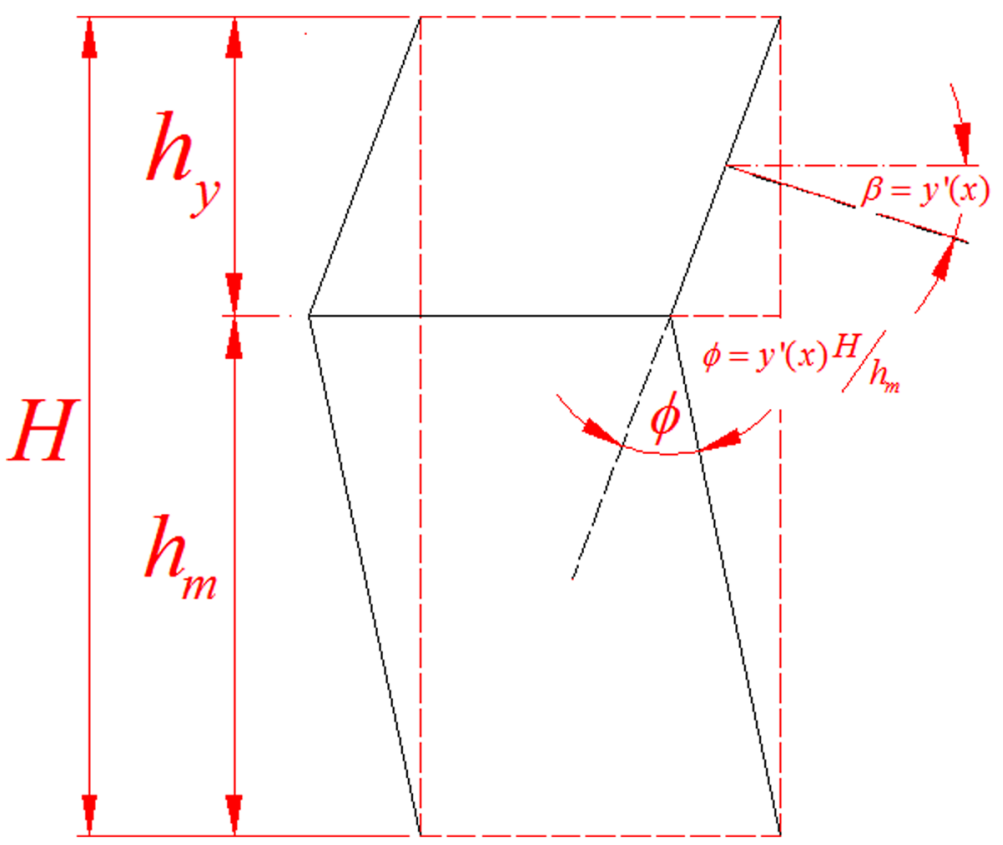

Fig. 3 Deformation relationship between fibre bundle and matrix

Then, equilibrium equations for fibre bundles can be written as (see Fig. 4):

$$
\left\{\begin{array}{l}
-M(x)+S_{0} x-\int_{0}^{x} q_{f}(x) \frac{h_{y}}{2} d s=M_{0} \\
S(x)+\int_{0}^{l} q_{f}(x) \sin \theta d s=S_{0} \\
q_{f}(x) \cos (\theta)=q_{m}(x)
\end{array}\right.
$$

where $M_{0}$ and $S_{0}$ are the moment and shear force at the end $(x=0)$, respectively; $M(x)$ and $S(x)$ are the moment and shear force of the beam element representing the bundle, respectively; $q_{m}(x)$ and $q_{f}(x)$ are the shear stress of matrix and fibre domains, respectively.

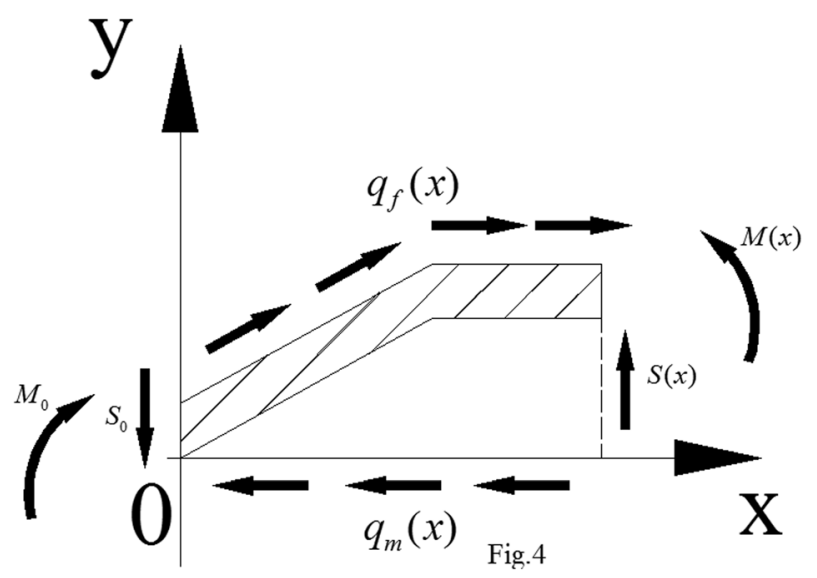

Fig. 4 Quarter of RVE of squeezed fibre bundles 
The moment of fibre can be described by:

$$
M(x)=\frac{E_{y 11} h_{y}^{3}}{12} y^{\prime \prime}(x)
$$

where $y(x)$ is the deflection shape equation of the beam element, $E_{y 11}$ is the elastic modulus of fibres along the 1 direction. Then, the equilibrium equations can be simplified as:

$$
\begin{gathered}
-\frac{E_{y 11} h_{y}^{3}}{12} y_{1}^{\prime \prime}(x)+S_{0} x-\int_{0}^{x} \frac{G_{m}}{\cos (\theta)}\left(1+\frac{h_{y} l}{2 r x}\right) y_{1}^{\prime}(x) \frac{h_{y}}{2} d s=M_{0},(0 \leq x<l), \\
-\frac{E_{y 11} h_{y}^{3}}{12} y_{2}^{\prime \prime}(x)+S_{0} x-\int_{0}^{x} \frac{G_{m}}{\cos (\theta)}\left(1+\frac{h_{y}}{2 r}\right) y_{2}^{\prime}(x) \frac{h_{y}}{2} d s=M_{0}, \quad(l \leq x<l+r) .
\end{gathered}
$$

The boundary conditions:

$$
\left\{\begin{array}{l}
y_{1},(0)=0 \\
y_{1}^{\prime}(0)=1
\end{array}\right.
$$

The Eq. (7a) cannot be solved analytically, so the forth-order approximation was used:

$$
\begin{aligned}
& y_{1}(x)=y_{1}(0)+y_{1}^{\prime}(0) x-\frac{3}{2} \frac{G_{m} h_{y}{ }^{2} l y_{1}{ }^{\prime}(0)+4 M_{0} r \cos \theta}{E_{y 11} h_{y}^{3} r \cos \theta} x^{2}- \\
& \frac{1}{2} \frac{2 G_{m} h_{y}^{2} r^{2} E_{y 11} \cos \theta y_{1}^{\prime}(0)-4 S_{0} E_{y 11} h_{y} r^{2} \cos ^{2} \theta-3 G_{m}^{2} h_{y}^{2} l^{2} y_{1}{ }^{\prime}(0)-12 G_{m} l r M_{0} \cos \theta}{E_{y 11}^{2} h_{y}^{4} r^{2} \cos ^{2} \theta} x^{3}+ \\
& \frac{3}{8} \frac{G_{m} \quad-3 E_{y 11} G_{m} h_{y}^{2} l r^{2} \cos \theta y_{1}^{\prime} l^{\prime} l_{1}^{3} y_{1}^{\prime}(0)+4 S_{0} E_{y 11} h_{y} l r^{2} \cos ^{2} \theta}{E_{y 11}^{3} h_{y}^{5} r^{3} \cos ^{3} \theta} x^{4}
\end{aligned}
$$

Using boundary conditions (8), the equations for moment $M_{1}(x)$ and shear force $S_{1}(x)$ can be solved $(0 \leq x<l)$ :

where

$$
M_{1}(x)=\frac{E_{y} h_{y}^{3}}{12} y_{1}^{\prime \prime}(x)=\frac{E_{y} h_{y}^{3}}{12}\left[3\left(Q_{1}+Q_{2} x\right)+\frac{9}{2} Q_{3} x^{2}\right] .
$$

$$
\left\{\begin{array}{l}
Q_{1}=\frac{G_{m} h_{y}{ }^{2} l+4 M_{0} r \cos \theta}{E_{y 11} h_{y}^{3} r \cos \theta}, \\
Q_{2}=\frac{2 G_{m} h_{y}^{2} r^{2} E_{y 11} \cos \theta-4 S_{0} E_{y 11} h_{y} r^{2} \cos ^{2} \theta-3 G_{m}^{2} h_{y}^{2} l^{2}-12 G_{m} l r M_{0} \cos \theta}{E_{y 11}^{2} h_{y}^{4} r^{2} \cos ^{2} \theta} \\
Q_{3}=\frac{G_{m}}{E_{y 11}^{3} h_{y}^{5} r^{3} \cos ^{3} \theta}\left(\begin{array}{l}
6 E_{y 11} G_{m} h_{y}^{2} l r^{2} \cos \theta-4 S_{0} E_{y 11} h_{y} l r^{2} \cos ^{2} \theta \\
-3 G_{m}^{2} h_{y}^{2} l^{3}+16 E_{y 11} M_{0} r^{3} \cos ^{2} \theta-12 G_{m} l^{2} r M_{0} \cos \theta
\end{array}\right) .
\end{array}\right.
$$

The shear force $S_{1}(x)$ can be obtained directly:

$$
S_{1}(x)=M_{1}^{\prime}(x)=\frac{E_{y} h_{y}^{3}}{4}\left[Q_{2}+3 Q_{3} x\right]
$$


The maximum axial stress is

$$
\sigma_{1 x}(x)= \pm \frac{M_{1}(x) d_{y}}{I_{z}}= \pm \frac{M_{1}(x) h_{y}}{4 I_{z}}=\frac{E_{y} h_{y}^{4}\left(3\left(Q_{1}+Q_{2} x\right)+\frac{9}{2} Q_{3} x^{2}\right)}{\left.\frac{11 b h_{y}^{3}}{2}+24 b h_{y} r^{2}+12 b h_{y}^{2} r\right)},(0 \leq x<l)
$$

where $b$ is the bundle's width.

The shear stress between the fibre bundle and the matrix is:

$$
\tau_{1 f}(x)=G_{m}\left(1+\frac{h_{y}}{2 r}\right) y_{1}^{\prime}(x)=G_{m}\left(1+\frac{h_{y}}{2 r}\right)\left[1-3 Q_{1} x+\frac{3}{2}\left(Q_{2} x^{2}+Q_{2} x^{3}\right)\right],(0 \leq x<l) .
$$

Equation (7b) can be solved exactly analytically as follows:

$$
y_{2}(x)=C_{1} e^{\omega x}+C_{2} e^{-\omega x}+\frac{4 S_{0} r \cos \theta}{G_{m} h_{y}\left(2 r+h_{y}\right)} x-\frac{4 M_{0} r \cos \theta}{G_{m} h_{y}\left(2 r+h_{y}\right)} \quad(l \leq x<l+r)
$$

Using the boundary conditions:

$$
\left\{\begin{array}{l}
y_{2}(l)=y_{1}(l) \\
y_{2}^{\prime}(l)=y_{1}^{\prime}(l) \\
y_{2}(L)=0 \\
y_{2}^{\prime}(L)=0
\end{array}\right.
$$

where $L=l+r$, the coefficients can be found as:

$$
\left\{\begin{array}{l}
C_{1}=\frac{e^{\omega L}-\omega L-1}{\omega\left(-2 e^{2 \omega L}+4 e^{\omega L}+\omega L e^{2 \omega L}-\omega L-2\right)} \\
C_{2}=\frac{-\omega L e^{2 \omega L}+e^{2 \omega L}-e^{\omega L}}{\omega\left(-2 e^{2 \omega L}+4 e^{\omega L}+\omega L e^{2 \omega L}-\omega L-2\right)} \\
S_{0}=\frac{\left(1-e^{\omega L}\right) G_{m} h_{y}\left(h_{y}+2 r\right)}{4 r \cos \theta\left(-2 e^{\omega L}+\omega L e^{\omega L}+2+\omega L\right)} \\
M_{0}=\frac{G_{m} h_{y}\left(h_{y}+2 r\right)\left(\omega L e^{2 \omega L}+\omega L-e^{2 \omega L}+1\right)}{4 \omega r \cos \theta\left(-2 e^{2 \omega L}+4 e^{\omega L}+\omega L e^{2 \omega L}-\omega L-2\right)}
\end{array}\right.
$$

where $\omega=\sqrt{\frac{6 G_{m}}{E_{y 11} h_{y}^{2} \cos \theta}\left[1+\frac{h_{y}}{\left(h_{y}+2 r\right) L}\right]}$.

So, the moment $M(x)$ and shear force $S(x)$ equations can be obtained for $l \leq x<L$ using Eqs. (15) and (17):

$$
\begin{gathered}
M_{2}(x)=\frac{E_{y 11} h_{y}^{3}}{12} y_{2}^{\prime \prime}(x)=\omega \frac{E_{y 11} h_{y}^{3}}{12} \frac{e^{\omega L}-\omega L-1-\omega L e^{2 \omega L-2 \omega x}+e^{2 \omega L-2 \omega x}-e^{\omega L-2 \omega x}}{-2 e^{2 \omega L}+4 e^{\omega L}+\omega L e^{2 \omega L}-\omega L-2} e^{\omega x} \\
S_{2}(x)=M_{2}^{\prime}(x)=\omega^{2} \frac{E_{y 11} h_{y}^{3}}{12} \frac{\omega L e^{2 \omega L-2 \omega x}-e^{2 \omega L-2 \omega x}+e^{\omega L-2 \omega x}+e^{\omega L}-\omega L-1}{-2 e^{2 \omega L}+4 e^{\omega L}+\omega L e^{2 \omega L}-\omega L-2} e^{\omega x}
\end{gathered}
$$


So that the maximum axial stress is:

$$
\begin{aligned}
& \sigma_{2 x}(x)= \pm \frac{M_{2}(x) d_{y}}{I_{z}}= \pm \frac{M_{2}(x) h_{y}}{4 I_{z}} \\
& = \pm \frac{\left(\omega E_{y 11} h_{y}^{3} \frac{e^{\omega L}-\omega L-1-\omega L e^{2 \omega L-2 \omega x}+e^{2 \omega L-2 \omega x}-e^{\omega L-2 \omega x}}{-2 e^{2 \omega L}+4 e^{\omega L}+\omega L e^{2 \omega L}-\omega L-2} e^{\omega x}\right) h_{y}}{\left(\frac{11}{2} b h_{y}^{3}+24 b h_{y} r^{2}+12 b h_{y}^{2} r\right)}(l \leq x<L)
\end{aligned}
$$

The shear stress between the fibre bundle and the matrix is:

$$
\begin{aligned}
& \tau_{2 f}(x)=G_{m}\left(1+\frac{h_{y}}{2 r}\right) y_{2}{ }^{\prime}(x)= \\
& G_{m}\left(1+\frac{h_{y}}{2 r}\right) \frac{e^{\omega x+\omega L}-(\omega L+1) e^{\omega x}+(\omega L-1) e^{2 \omega L-\omega x}+e^{\omega L-\omega x}-e^{2 \omega L}+2 e^{\omega L}-1}{-2 e^{2 \omega L}-4 e^{\omega L}+\omega L e^{2 \omega L}-\omega L-2} \quad(l \leq x<L)
\end{aligned}
$$

\subsection{Mesoscale Mechanical Model of Deflected Fibre Bundles}

Changing from the plane x-o-y (Fig. 4) to x-o-z (Fig. 5), another RVE can be introduced to analyse the in-plane deflection of fibre bundles caused by needle-punching.

Based on the RVE geometry, the relationship for deformation of the fibre bundle and the matrix can be obtained as:

$$
\left\{\begin{array}{l}
\gamma_{z m}=\frac{h_{z f}}{h_{z m}} z^{\prime}(x)=\frac{h_{z f}}{h_{z m}} r_{m} \cos \alpha z^{\prime}(\alpha) \\
h_{z f}=h_{z y}+h_{z m}
\end{array}\right.
$$

where $h_{z y}, h_{z m}$ is the height of fibre bundle and matrix, respectively, $\gamma_{z m}$ is the shear strain of matrix.

So, shear stress of the matrix is:

$$
q_{z m}(\alpha)=G_{m} \gamma_{z m}=G_{m} \frac{h_{z f}}{h_{z m}} r_{m} z^{\prime}(\alpha) \cos \alpha
$$
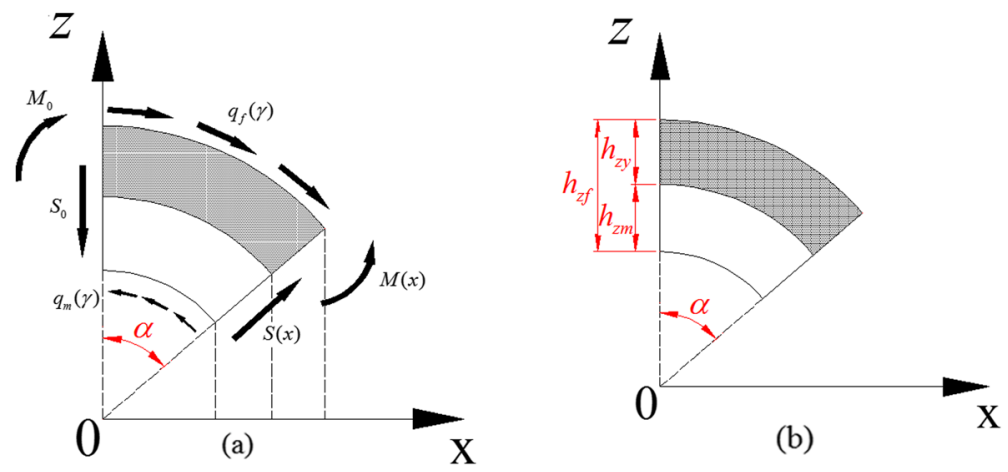

(b)

Fig. 5 RVE model of deflected fibre bundles: a analysis of internal force; (b) shape parameters 
The differential equations of equilibrium in a polar coordinate system can be written

$$
\left\{\begin{array}{l}
-M_{z}(\alpha)+S_{z 0} r_{f} \sin \alpha-\int_{0}^{\gamma} q_{z f}(\alpha) \frac{h_{z f}}{2} r_{f} \cos \alpha d \alpha=M_{z 0} \\
S_{z}(\alpha)-\int_{0}^{\gamma} q_{z f}(\alpha) \cos \alpha d \alpha=S_{z 0} \\
\int_{0}^{\gamma} q_{z f}(\alpha) r_{f} \gamma d \alpha=\int_{0}^{\gamma} q_{z m}(\alpha) r_{m} \gamma d \alpha \\
r_{f}=r_{m}+\frac{1}{2} h_{z f}+\frac{1}{2} h_{z m}
\end{array}\right.
$$

where $M_{y}(\alpha)=\frac{E_{z} h_{z f}^{3}}{12} z^{\prime \prime}(x)=-\frac{E_{z} h_{z f}^{3}}{12} r_{f} \sin (\alpha) z^{\prime \prime}(\alpha)$.

The shear stress in fibre bundle has the following form:

$$
q_{z f}(\alpha)=\frac{G_{m} h_{y}}{h_{m}} r_{m} \cos \alpha\left[1-\frac{h_{z f}+h_{z m}}{2 r_{f}}\right] z^{\prime}(\alpha)+c_{1} \quad\left(c_{1}=0\right)
$$

Equation (24) after simplification is:

$$
z^{\prime \prime}(\alpha)-\left[\frac{3 G_{m}\left(2 r_{f}-h_{z f}-h_{z m}\right)}{E_{z} h_{z f} h_{z m} r_{f}}\right] \frac{\cos ^{2} \alpha}{\sin \alpha} z^{\prime}(\alpha)=\frac{12\left(M_{z 0}-S_{z 0} r_{f} \sin \alpha\right)}{E_{z} h_{z f}^{3} r_{f} \sin \alpha}
$$

It has the following solution:

$$
z(\alpha)=C_{z 1} e^{\lambda \alpha}+C_{z 2} e^{-\lambda \alpha}+\frac{4 h_{z m} M_{z 0}}{G_{m} h_{z f}^{2}\left(2 r_{f}-h_{z f}-h_{z m}\right) \cos ^{2} \alpha}-\frac{4 h_{z m} S_{z 0} r_{f} \sin \alpha}{G_{m} h_{z f}^{2}\left(2 r_{f}-h_{z f}-h_{z m}\right) \cos ^{2} \alpha}
$$

where $\lambda=\sqrt{\frac{3 G_{m}\left(2 r_{f}-h_{z f}-h_{z m}\right) \cos ^{2} \alpha}{E_{z} h_{f f} h_{z m} r_{f} \sin \alpha}}$.

Then, from Eq. (28) and the boundary conditions (29), parameters of Eq. (27) can be obtained as Eq. (30):

$$
\begin{gathered}
z^{\prime}(\alpha)=\lambda C_{z 1} e^{\lambda \alpha}-\lambda C_{z 2} e^{-\lambda \alpha}+ \\
\frac{8 M_{z 0} h_{z m} \sin \alpha}{G_{m} h_{z f}^{2}\left(2 r_{f}-h_{z f}-h_{z m}\right) \cos ^{3} \alpha}-\frac{4 h_{z m} S_{z 0} r_{f}\left(1+\sin ^{2} \alpha\right)}{G_{m} h_{z f}^{2}\left(2 r_{f}-h_{z f}-h_{z m}\right) \cos ^{3} \alpha} \\
\qquad \begin{array}{l}
z(0)=0 \\
z^{\prime}(0)=1 \\
z(\gamma)=0 \\
z^{\prime}(\gamma)=0
\end{array} \\
\left\{\begin{array}{l}
C_{z 1}=\frac{e^{\lambda \gamma-\lambda \sin \gamma \cos \gamma+\cos ^{2} \gamma-2}}{D(\lambda, \gamma)} \\
C_{z 2}=-e^{\lambda \gamma} \frac{-2 e^{\lambda \gamma}+\cos ^{2} \gamma e^{\lambda \gamma}+\lambda \sin \gamma \cos \gamma e^{\lambda \gamma}+1}{D(\lambda, \gamma)} \\
M_{z 0}=\frac{2-\cos ^{2} \gamma+\lambda \sin \gamma \cos ^{2}-2 e^{2 \lambda \gamma}+\cos ^{2} \gamma e^{2 \lambda \gamma}+\lambda \sin \gamma \cos \gamma e^{2 \lambda \gamma}}{A \cdot D(\lambda, \gamma)} \\
S_{z 0}=\frac{-2 \lambda \cos ^{3} \gamma e^{\lambda \gamma}-2 \sin \gamma e^{2 \lambda \gamma}+\lambda \cos \gamma+2 \sin \gamma+\lambda \cos \gamma e^{2 \lambda \gamma}}{B \cdot D(\lambda, \gamma)}
\end{array}\right.
\end{gathered}
$$


where

$$
\begin{aligned}
& D(\lambda, \gamma)=-\lambda^{2} \cos \gamma \sin \gamma+2 \lambda e^{\lambda \gamma}-2 \lambda e^{2 \lambda \gamma}+\lambda \cos ^{2} \gamma e^{2 \lambda \gamma}+2 \lambda \cos ^{3} \gamma e^{\lambda \gamma}+2 \sin \gamma e^{2 \lambda \gamma} \\
& -\lambda \cos \gamma+\lambda^{2} e^{2 \lambda \gamma} \sin \gamma \cos \gamma-2 \lambda+\lambda \cos ^{2} \gamma-2 \sin \gamma-\lambda e^{2 \lambda \gamma} \cos \gamma, \\
& A=\frac{4 h_{z m}}{G_{m} h_{z f}^{2}\left(2 r_{f}-h_{z f}-h_{z m}\right)}, B=\frac{4 h_{z m} r_{f}}{G_{m} h_{z f}^{2}\left(2 r_{f}-h_{z f}-h_{z m}\right)} .
\end{aligned}
$$

Please check displayed equation below if presented correctly.

So, the full form of deflection equation for the fibre bundles is solved:

$$
\begin{aligned}
z(\alpha) & =C z_{1} e^{\lambda \alpha}+C z_{2} e^{-\lambda \alpha}+\frac{4 h_{z m} M_{z 0}}{G_{m} h_{z f}^{2}\left(2 r_{f}-h_{z f}-h_{z m}\right) \cos ^{2} \alpha}-\frac{4 h_{z m} S_{z 0} r_{f} \sin \alpha}{G_{m} h_{z f}^{2}\left(2 r_{f}-h_{z f}-h_{z m}\right) \cos ^{2} \alpha} \\
& =\frac{S_{2}}{S_{1} \cos ^{2} \alpha}
\end{aligned}
$$

where,

$$
\left\{\begin{aligned}
S_{1} & =-\lambda^{2} \sin \gamma \cos \gamma+2 \lambda e^{\lambda \gamma}-2 \lambda e^{2 \lambda \gamma}+\lambda \cos ^{2} \gamma e^{2 \lambda \gamma}+2 \lambda \cos ^{3} \gamma e^{\lambda \gamma}+2 \sin \gamma e^{2 \lambda \gamma} \\
& -\lambda \cos \gamma+\lambda^{2} \sin \gamma \cos \gamma e^{2 \lambda \gamma}-2 \lambda+\lambda \cos ^{2} \gamma-2 \sin \gamma-\lambda \cos \gamma e^{2 \lambda \gamma} \\
S_{2} & =\left(\lambda \sin \gamma \cos \gamma+2-\cos ^{2} \gamma-2 e^{2 \lambda \gamma}+\lambda \sin \gamma \cos \gamma e^{2 \lambda \gamma}+e^{2 \lambda \gamma} \cos ^{2} \gamma\right) \\
& +\left(2 e^{2 \lambda \gamma} \sin \gamma-\lambda e^{2 \lambda \gamma} \cos \gamma+2 \lambda \cos ^{3} \gamma e^{\lambda \gamma}-2 \sin \gamma-\lambda \sin \gamma\right) \sin \alpha \\
& +\left(-\lambda e^{\lambda \alpha} \sin \gamma \cos \gamma+2 e^{\lambda(2 \gamma-\alpha)}-e^{\lambda(\gamma-\alpha)}+e^{\lambda(\alpha+\gamma)}-e^{\lambda(2 \gamma-\alpha)} \cos ^{2} \gamma-\right. \\
& \left.2 e^{\lambda \alpha}+e^{\lambda \alpha} \cos ^{2} \gamma\right) \cos ^{2} \alpha-\lambda \sin \gamma \cos \gamma e^{\lambda(2 \gamma-\alpha)}
\end{aligned}\right.
$$

The moment and shear force of the fibre bundle are:

$$
\left\{\begin{array}{l}
M_{y f}(\alpha)=\frac{E_{y 11} h_{z f}^{3}}{12} z^{\prime \prime}(x)=-\frac{E_{y 11} h_{z f}^{3}}{12} r_{f} \sin \alpha z^{\prime \prime}(\alpha)=\frac{E_{y 11} h_{z f}^{3} r_{f} S_{3}}{12 S_{1} \cos ^{4} \alpha} \\
S_{z f}(\alpha)=M_{z}^{\prime}(\alpha)=\frac{S_{4}}{S_{1} \cos ^{5} \alpha}-\frac{S_{5}}{S_{1} \cos ^{3} \alpha}
\end{array}\right.
$$

where

$$
\begin{aligned}
& \int S_{3}=-12+6 \lambda \cos \gamma \sin \alpha+\lambda^{2} e^{\lambda(\gamma-\alpha)} \cos ^{4} \alpha-12 \lambda e^{\lambda \theta} \cos ^{3} \gamma \sin \alpha+ \\
& 12 \sin \gamma \sin \alpha+8 \cos ^{2} \alpha+6 \cos ^{2} \gamma-6 \lambda \sin \gamma \cos \gamma-6 \lambda e^{2 \lambda \gamma} \sin \gamma \cos \gamma \\
& +6 \lambda e^{2 \lambda \gamma} \cos \gamma \sin \alpha-12 e^{2 \lambda \gamma} \sin \gamma \sin \alpha-6 e^{2 \lambda \gamma} \cos ^{2} \gamma+12 e^{2 \lambda \gamma}- \\
& 2 \lambda^{2} e^{2 \lambda \gamma-\lambda \alpha} \cos ^{4} \alpha-\lambda^{2} e^{\lambda \alpha+\lambda \gamma} \cos ^{4} \alpha-\lambda e^{2 \lambda \gamma} \cos \gamma \sin \alpha \cos ^{2} \alpha+ \\
& \lambda^{3} e^{2 \lambda \gamma-\lambda \alpha} \sin \gamma \cos \gamma \cos ^{4} \alpha+\lambda^{2} e^{2 \lambda \gamma-\lambda \alpha} \cos ^{2} \gamma \cos ^{4} \alpha-\lambda \cos \gamma \sin \alpha \cos ^{2} \alpha \\
& -\lambda^{2} \cos ^{2} \gamma e^{\lambda \alpha} \cos ^{4} \alpha+2 \lambda e^{\lambda \gamma} \cos ^{3} \gamma \sin \alpha \cos ^{2} \alpha+\lambda^{3} e^{\lambda \alpha} \sin \gamma \cos \gamma \cos ^{4} \alpha \\
& +2 e^{2 \lambda \gamma} \sin \gamma \sin \alpha \cos ^{2} \alpha+4 \lambda e^{2 \lambda \gamma} \sin \gamma \cos \gamma \cos ^{2} \alpha+4 \lambda \sin \gamma \cos \gamma \cos ^{2} \alpha \\
& +2 \lambda^{2} e^{\lambda \alpha} \cos ^{4} \alpha+4 e^{2 \lambda \gamma} \cos ^{2} \gamma \cos ^{2} \alpha-2 \sin \gamma \sin \alpha \cos ^{2} \alpha \\
& -8 e^{2 \lambda \gamma} \cos ^{2} \alpha-4 \cos ^{2} \gamma \cos ^{2} \alpha \\
& S_{4}=48 \sin \alpha+48 \lambda e^{\lambda \gamma} \cos ^{3} \gamma+18 \lambda \cos \gamma \cos ^{2} \gamma+24 e^{2 \lambda \gamma} \cos ^{2} \gamma \sin \alpha+ \\
& 8 \cos ^{2} \gamma \sin \alpha \cos ^{2} \alpha-2 \lambda^{3} e^{\lambda(2 \gamma-\alpha)} \cos ^{5} \alpha+\lambda^{3} e^{\lambda(\alpha+\gamma)} \cos ^{5} \alpha+\lambda^{3} e^{\lambda(\gamma-\alpha)} \cos ^{5} \alpha \\
& -2 \lambda^{3} e^{\lambda \alpha} \cos ^{5} \alpha-36 \lambda e^{\lambda \gamma} \cos ^{3} \gamma \cos ^{2} \alpha+24 \lambda \sin \gamma \cos \gamma \sin \alpha+ \\
& 24 \lambda e^{2 \lambda \gamma} \sin \gamma \sin \alpha+18 \lambda e^{2 \lambda \gamma} \cos \gamma \cos ^{2} \alpha-24 \lambda \cos \gamma-2 \sin \gamma \cos ^{4} \alpha- \\
& 16 \sin \alpha \cos ^{2} \alpha-48 \sin \gamma-24 \cos ^{2} \gamma \sin \alpha+40 \sin \gamma \cos ^{2} \alpha-48 e^{2 \lambda \gamma} \sin \alpha- \\
& 8 e^{2 \lambda \gamma} \cos ^{2} \gamma \sin \alpha \cos ^{2} \alpha+\lambda^{4} e^{\lambda(2 \gamma-\alpha)} \sin \gamma \cos \gamma \cos ^{5} \alpha-36 e^{2 \lambda \gamma} \sin \gamma \cos ^{2} \alpha- \\
& 24 \lambda e^{2 \lambda \gamma} \cos \gamma+48 e^{2 \lambda \gamma} \sin \gamma \\
& S_{5}=\lambda \cos \gamma \cos ^{2} \alpha+8 \lambda \sin \gamma \cos \gamma \sin \alpha-\lambda^{3} e^{\lambda \alpha} \cos ^{2} \gamma \cos ^{3} \alpha+ \\
& \lambda^{4} e^{\lambda \alpha} \sin \gamma \cos \gamma \cos ^{3} \alpha-2 \lambda \cos \gamma+4 e^{2 \lambda \gamma} \sin \gamma-2 e^{2 \lambda \gamma} \sin \gamma \cos ^{2} \alpha- \\
& 2 \lambda e^{2 \lambda \gamma} \cos \gamma+\lambda e^{2 \lambda \gamma} \cos \gamma \cos ^{2} \alpha-2 \lambda e^{\lambda \gamma} \cos ^{3} \gamma \cos ^{2} \alpha+8 \lambda e^{2 \lambda \gamma} \sin \gamma \\
& \cos \gamma \sin \alpha-\lambda^{3} e^{\lambda(2 \gamma-\alpha)} \cos ^{2} \gamma \cos ^{3} \alpha+4 \lambda e^{\lambda \gamma} \cos ^{3} \gamma-16 e^{2 \lambda \gamma} \sin \alpha
\end{aligned}
$$


Using geometric neutral surface considerations of a circular beam (Fig. 6):

$$
a=R-\rho=R-\frac{h}{\ln \frac{R_{0}}{R_{1}}}
$$

Together with the Taylor series expansion omitted higher order term [24-26]:

$$
a=\frac{h^{2}}{12 R}
$$

The axial stress of the beam element can be presented as:

$$
\sigma_{x}(\alpha)=\frac{M_{y f}(\alpha) z}{a b h(\rho+z)}
$$

The shear stress of the fibre bundle has the following form:

$$
\tau_{z f}(\alpha)=q_{z f}(\alpha)=\frac{G_{m} h_{y}}{h_{m}} r_{m} \cos \alpha\left[1-\frac{h_{z f}+h_{z m}}{2 r_{f}}\right] z^{\prime}(\alpha)
$$

where, the full form of $z^{\prime}(\alpha)$ is given:

$$
z^{\prime}(\alpha)=\frac{S_{6}}{S_{1} \cos ^{3} \alpha}
$$

where,

$$
\begin{aligned}
& S_{6}=-2 e^{2 \lambda \gamma} \sin \gamma \cos ^{2} \alpha+4 \sin \alpha+4 \lambda e^{\lambda \gamma} \cos ^{3} \gamma-2 \lambda e^{\lambda(2 \gamma-\alpha)} \cos ^{3} \alpha+ \\
& \lambda e^{\lambda(\alpha+\gamma)} \cos ^{3} \alpha+\lambda \cos \gamma \cos ^{2} \alpha-2 \lambda e^{\lambda \alpha} \cos ^{3} \alpha+\lambda e^{\lambda(\gamma-\alpha)} \cos ^{3} \alpha+ \\
& 2 e^{2 \lambda \gamma} \cos ^{2} \gamma \sin \alpha-2 \lambda \cos \gamma-\lambda^{2} e^{\lambda \alpha} \sin \gamma \cos ^{3} \gamma \cos ^{3} \alpha+\lambda e^{\lambda \alpha} \cos ^{3} \alpha+ \\
& \lambda^{2} e^{\lambda(2 \gamma-\alpha)} \sin \gamma \cos \gamma \cos ^{3} \alpha-2 \lambda e^{\lambda \gamma} \cos ^{3} \gamma \cos ^{2} \alpha+\lambda e^{2 \lambda \gamma} \cos ^{2} \gamma \cos ^{2} \alpha+ \\
& \lambda e^{\lambda(2 \gamma-\alpha)} \cos ^{2} \gamma \cos ^{3} \alpha+2 \lambda \sin \gamma \cos \gamma \sin \alpha+2 \lambda e^{2 \lambda \gamma} \sin \gamma \cos \gamma \sin \alpha- \\
& 2 \cos ^{2} \gamma \sin \alpha-4 \sin \gamma+2 \sin \gamma \cos ^{2} \alpha-4 e^{2 \lambda \gamma} \sin \alpha-2 \lambda e^{2 \lambda \gamma} \cos \gamma+4 e^{2 \lambda \gamma} \sin \gamma
\end{aligned}
$$
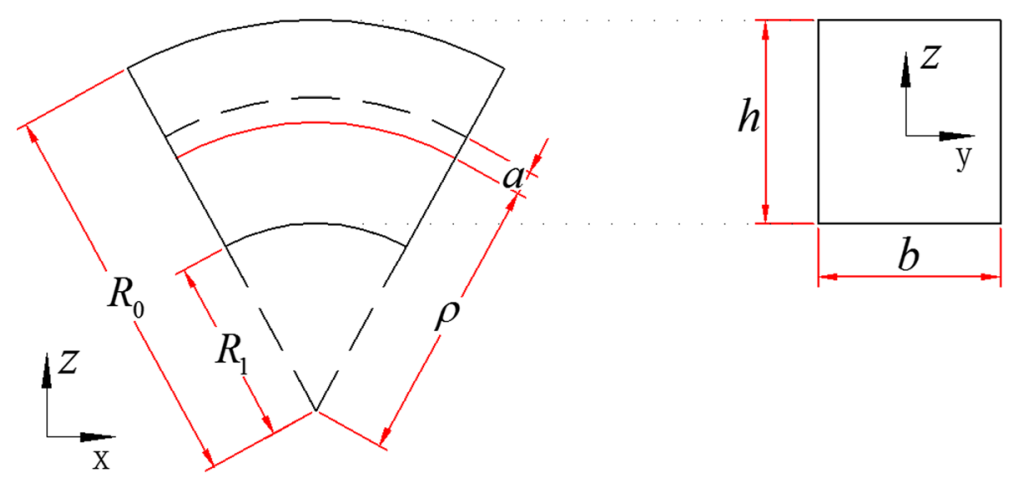

Fig. 6 Section parameters 


\section{Conclusion}

The theoretical bending model was suggested for UD NP C/Cs to analyse the stress distribution of fibre bundles after the application of the needle-punching technology. The suggested analytical approach can be used for predictions of stress distribution in fibre bundles for design and optimization of $\mathrm{C} / \mathrm{Cs}$.

Acknowledgements This work is partially supported by the National Natural Science Foundation of China (11872205, 11272147), SKL Open Fund (MCMS-0218G01), Foundation of National Scholarship of China (201706830073), Priority Academic Program Development of Jiangsu Higher Education Institutions.

\section{References}

1. Li, X., Yu, S., Li, Y.P., Wu, Q., Li, Z., Xiao, T., et al.: Effect of pre-fatigue on bending behavior of 2.5D C/C composites. Mater. Sci. Eng. A. 682, 290-295 (2017)

2. Li, D.S., Duan, H.W., Wang, W., Ge, D.Y., Jiang, L., Yao, Q.Q.: Strain rate and temperature effect on mechanical properties and failure of 3D needle-punched carbon/carbon composites under dynamic loading. Compos. Struct. 172, 229-241 (2017)

3. Alipour, M.M.: An analytical approach for bending and stress analysis of cross/angle-ply laminated composite plates under arbitrary non-uniform loads and elastic foundations. Arch. Civ. Mech. Eng. 16, 193-210 (2016)

4. Zhang, M.Y., Su, Z.A., Li, J.L., Huang, Q.Z.: Bending properties and fracture mechanism of C/C composites with high density preform. Trans. Nonferrous Metals Soc. China. 21, 1795-1800 (2011)

5. Zhang, M.Y., Su, Z., Xie, Z., Chen, J., Huang, Q.: Microstructure of pyrocarbon with chemical vapor infiltration. Procedia Eng. 27, 847-854 (2011)

6. Li, D.S., Yao, Q., Jiang, N., Jiang, L.: Bend properties and failure mechanism of a carbon/carbon composite with a 3D needle-punched preform at room and high temperatures. New Carbon Mater. 31, 437-444 (2016)

7. Li, D.S., Fang, D.N., Zhang, G.B., Hu, H.: Effect of temperature on bending properties and failure mechanism of three-dimensional braided composite. Mater. Des. 41, 167-170 (2012)

8. Li, D.S., Li, J.L., Chen, L., Lu, Z.X., Fang, D.N.: Finite element analysis of mechanical properties of 3D four-directional rectangular braided composites part 1: microgeometry and 3D finite element model. Appl. Compos. Mater. 17, 373-387 (2010)

9. Zhang, Y., Lu, Z., Yang, Z., Zhang, D., Shi, J., Yuan, Z., Liu, Q.: Compression behaviors of carbon-bonded carbon fibre composites: experimental and numerical investigations. Carbon. 116, 398-408 (2017)

10. Zhang, Y., Lu, Z., Yang, Z.: An interface model of the fiber pullout process of the carbon nanotubes hybridized carbon fiber composites. In: 17th European conference on composite materials. ECCM, Munich (2016)

11. Cai, Y., Fan, S., Yin, X., Zhang, L., Cheng, L., Wang, Y.: Microstructures and mechanical properties of three-dimensional ceramic filler modified carbon/carbon composites. Ceram. Int. 40, 399-408 (2014)

12. Cai, Y., Yin, X., Fan, S., Zhang, L., Cheng, L.: Tribological behavior of three-dimensional needled ceramic modified carbon/carbon composites in seawater conditions. Compos. Sci. Technol. 87, 50-57 (2013)

13. Cai, Y., Fan, S., Liu, H., Zhang, L., Cheng, L., Dong, B., Jiang, J.: Microstructures and improved wear resistance of 3D needled $\mathrm{C} / \mathrm{SiC}$ composites with graphite filler. Compos. Sci. Technol. 69, 2447-2453 (2009)

14. Mouritz, A.P.: Compression properties of z-pinned composite laminates. Compos. Sci. Technol. 67, 31103120 (2007)

15. Belingardi, G., Cavatorta, M.P., Frasca, C.: Bending fatigue behavior of glass-carbon/epoxy hybrid composites. Compos. Sci. Technol. 66, 222-232 (2006)

16. Patel, N.P., Sharma, D.S.: Bending of composite plate weakened by square hole. Int. J. Mech. Sci. 94-95, 131-139 (2015)

17. Lacoste, M., Lacombe, A., Joyez, P.: Carbon/Carbon extendible nozzles. Acta Astronaut. 50, 357-367 (2002)

18. Turner, P., Liu, T., Zeng, X.: Collapse of 3D orthogonal woven carbon fibre composites under in-plane tension/compression and out-of-plane bending. Compos. Struct. 142, 286-297 (2016) 
19. Liu, W.: Element rigidity matrix and coordinate transformation matrix of space circular arc curved beam. Journal of Wuhan Institute of Water Transportation. Engineering. 27, 97-106 (1985)

20. Dietrich, S., Gebert, J.-M., Stasiuk, G., Wanner, A., Weidenmann, K.A., Deutschmann, O., Tsukrov, I., Piat, R.: Microstructure characterization of CVI-densified carbon/carbon composites with various fibre distributions. Compos. Sci. Technol. 72, 1892-1900 (2012)

21. Koricho, E.G., Belingardi, G.: An experimental and finite element study of the transverse bending behaviour of CFRP composite T-joints in vehicle structures. Composites Part B. 79, 430-443 (2015)

22. Alshahrani, H., Hojjati, M.: A theoretical model with experimental verification for bending stiffness of thermosetting prepreg during forming process. Compos. Struct. 166, 136-145 (2017)

23. Ropers, S., Kardos, M., Osswald, T.A.: A thermo-viscoelastic approach for the characterization and modeling of the bending behavior of thermoplastic composites. Compos. Part A. 90, 22-32 (2016)

24. D’Ottavio, M., Dozio, L., Vescovini, R.: Bending analysis of composite laminated and sandwich structures using sublaminate variable-kinematic Ritz models. Compos. Struct. 155, 45-62 (2016)

25. Sobuz, H.R., Ahmed, E., Sutan, N.M., Hasan, N.M., Uddin, M.A., Uddin, M.J.: Bending and timedependent responses of RC beams strengthened with bonded carbon fibre composite laminates. Constr. Build. Mater. 29, 597-611 (2012)

26. Margossian, A., Bel, S., Hinterhoelzl, R.: Bending characterisation of a molten unidirectional carbon fibre reinforced thermoplastic composite using a dynamic mechanical analysis system. Compos. Part A. 77, 154 163 (2015) 